\title{
TRATAMIENTO DE UNA REABSORCIÓN EXTERNA INFLAMATORIA CON TRIÓXIDO DE MINERAL AGREGADO: REPORTE DE UN CASO
}

\author{
Humberto Ferreira Arquez \\ Odontólogo U. de Cartagena, Especialista en Endodoncia U. Santo Tomás, \\ Docente Facultad de Medicina U. de Pamplona
}

Correo electrónico: humfear@yahoo.com

\begin{abstract}
RESUMEN
Paciente masculino de 9 años de edad, sin antecedentes médicos que contribuyan al caso. En el examen clínico y radiográfico se observó una extensa y progresiva reabsorción radicular externa inflamatoria, una extensa lesión periapical, una bolsa periodontal y movilidad dentaria grado 2 a nivel del 21. Cinco meses atrás, el paciente sufrió trauma dental (avulsión), ese incisivo, fue mantenido en condiciones secas (envuelto en un pañuelo), reimplantado, reposicionado y ferulizado por odontólogo general dos horas después del trauma. Seguido a la preparación biomecánica se realizaron recambios de hidróxido de calcio cada cuatro semanas durante 6 meses; las características clínico patológicas mostraron una resolución parcial del proceso (disminución de la lesión periapical, pero persistía la pérdida ósea, la reabsorción radicular externa, la movilidad dentaria grado 2; y el defecto periodontal). Se decidió obturar completamente el conducto radicular con MTA blanco, los exámenes radiográficos seis meses después mostraron cambios en la densidad - trabeculado del hueso alveolar y la lámina ósea alrededor de la superficie radicular, no fue encontrada bolsa periodontal, la movilidad del diente fue considerada normal (grado 1), con subsecuente detención del proceso de reabsorción. Radiografias a los 24- 48- 54 meses mostraron una adecuada reparación de la reabsorción. Clínicamente el diente fue asintomático. El propósito de este artículo es reportar la reparación y detención de una extensa reabsorción radicular externa inflamatoria mediante el uso de MTA. [Ferreira H. Tratamiento con Trióxido de Mineral Agregado en una reabsorción externa inflamatoria: reporte de caso. Ustasalud 2009; 8: 53 - 59 ]
\end{abstract}

Palabras clave: Reabsorción externa inflamatoria, Trióxido de mineral agregado, Hidróxido de calcio.

\section{TREATMENT OF AN EXTERNAL INFLAMMATORY RESORPTION WITH MINERAL TRIOXIDE AGGREGATE: A CASE REPORT}

\begin{abstract}
A 9-year old male, the patient's medical histories were all noncontributory. A clinical and radiographic examination revealed an extensive and progressive external inflamatory, extensive periapical lesion, periodontal pocket, grade II mobility at level of the 21 Five months behind the patient suffered dental trauma (avulsion), that incisive, was stored under dry conditions (wrapped in a handkerchief), replanted ,repositioned and splinted by general dentist 2 hours after trauma. Following biomechanical preparation, a dressing of calcium hydroxide was applied and replaced every 4 weeks over a period of 6 months; The clinical-pathological picture demonstrated partial resolution (decrease of periapical lesion, but it persisted: the loss of bony, the external root resorption, the grade II mobility, and the defect periodontal) decides to filling the root canal completely with MTA white, The radiographic examinations 6 mouth after revealed changes in the density - trabeculade of the alveolar bone, and the lamina bony around the root surface, no periodontal pocket was found, the mobility tooth was considered normal (grade I), with subsequent arrest of the process resorptive. Radiographs at $24,48,54$ months showed adequate repair of the resorption. Clinically, the tooth was asymptomatic. The aim of this article is report the repair and arrest of an extensive external inflammatory, by means of the use of MTA.
\end{abstract}

Key words: External inflammatory resoprtion, Mineral trioxide aggregate, Calcium hydroxide.

Recibido para publicación: 20 de enero de 2009. Aceptado para publicación: 15 de junio de 2009.

\section{INTRODUCCIÓN}

La reabsorción externa inflamatoria y la reabsorción por reemplazo son complicaciones resultado del trauma dental. Cuando los dientes luxados o avulsionados son reimplantados, el daño del ligamento periodontal, la pérdida del precemento, de los cementoblastos y de los restos epiteliales de Malassez ocasionan una superficie radicular denudada, iniciándose una reabsorción quimiotáctica del tejido duro. Los macrófagos y osteoclastos remueven el ligamento periodontal y el cemento dañado. El desarrollo de la reabsorción externa inflamatoria y de la reabsorción por reemplazo están directamente relacionadas con el daño al periodonto al tiempo del trauma así como la presencia de bacterias dentro del conducto radicular y dentro de los túbulos dentinales. ${ }^{1-4}$ 
Las condiciones favorables para reimplantar un diente incluyen la preservación de la vitalidad del ligamento periodontal, la integridad del cemento y una mínima contaminación bacteriana así se podrá esperar que el pronóstico sea bueno y que exista una alta tasa de supervivencia. Esas condiciones son directamente proporcionales al tiempo extraoral, medio de almacenamiento (transporte) y alteraciones de la superficie radicular. La probabilidad de daño de la superficie radicular se incrementa cuando el diente avulsionado no se reimplanta inmediatamente o si no es almacenado en un adecuado medio. Estas situaciones, invariablemente, inician la necrosis del tejido pulpar y del ligamento periodontal. La presencia de un remanente necrótico de ligamento periodontal estimula la ocurrencia de reabsorción radicular externa, la cual es la mayor causa de pérdida de los dientes reimplantados. ${ }^{5-20}$

Este reporte de caso describe la subsecuente detención de una extensa y progresiva reabsorción radicular externa inflamatoria, la resolución de una extensa lesión periapical en un diente avulsionado que fue reimplantado y reposicionado después de un tiempo extraoral de dos horas en un medio seco de almacenamiento. El tratamiento consistió en una apropiada terapia quimio-mecánica de conductos, dressing de hidróxido de calcio y obturación completa del conducto radicular con Trióxido de Mineral Agregado (MTA).

\section{REPORTE DE CASO}

En noviembre del 2003, un niño de 9 años de edad sufre una lesión traumática en el incisivo central superior izquierdo (21) al caerse de su bicicleta. El diente sufre avulsión, fue mantenido bajo condiciones secas (envuelto en un pañuelo) desde el momento en que sucede el trauma hasta que es atendido en un centro hospitalario. El diente avulsionado fue reimplantado y reposicionado por un odontólogo general, dos horas después del trauma. El paciente fue medicado con Amoxicilina de 500mg cada 8 horas por 5 días y Acetaminofen de $500 \mathrm{mg}$ cada 8 horas por 5 días. El diente fue ferulizado con una férula rígida fabricada en resina de fotocurado y mantenida en posición durante un mes. $\mathrm{Al}$ cabo de ese tiempo, la férula fue removida y se citó al paciente a los 15 días para realizar el tratamiento de conductos del diente 21; sin embargo, el paciente no asiste a esa cita.

Cinco meses después los padres del paciente consultan al ortodoncista para valoración y posterior inicio del tratamiento. Éste es informado por la madre del niño acerca del accidente. Clínicamente, observa que el diente 21 presenta movilidad anormal por lo que lo remite a consulta de valoración con un endodoncista.
Se observa que el diente 21 se encontraba vestibulizado y presentaba movilidad dentaria grado 2; los tejidos blandos circundantes se observaban normales sin evidencia de cambios de color en la corona del diente, presentaba dolor a la percusión, respondía negativamente a las pruebas de sensibilidad pulpar, al sondaje se encontró una bolsa periodontal de aproximadamente $8 \mathrm{~mm}$ de profundidad en las superficies mesial y distal, y de $6 \mathrm{~mm}$ de profundidad en las superficies vestibular y palatina.

En el examen radiográfico, se observó una avanzada y extensa reabsorción radicular externa inflamatoria, lesión radiolúcida de aproximadamente $14 \mathrm{x}$ $13 \mathrm{~mm}$ de diámetro, pérdida de hueso alveolar, pérdida de la continuidad del ligamento periodontal (Figura 1). El pronóstico fue considerado: malo. El propósito del tratamiento fue encaminado en detener la reabsorción, promover la reparación y el mantenimiento del hueso alveolar en procura de que el diente permaneciera en boca el mayor tiempo posible para facilitar la posterior realización de una prótesis.

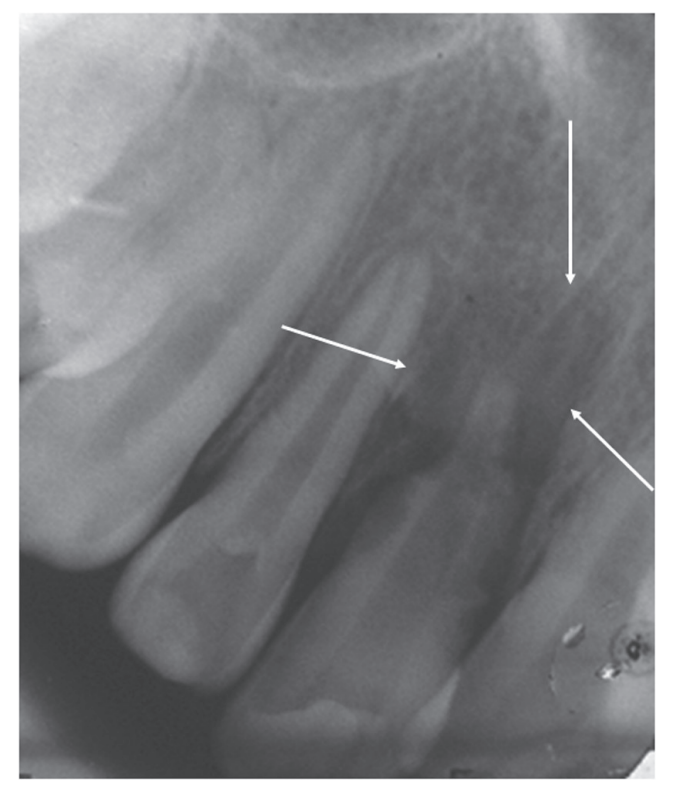

Figura 1. Las flechas señalan la extensión de la lesión radiolúcida. Nótese la perdida ósea en mesial y distal del 21 e igualmente la extensión y progresión del proceso reabsortivo a nivel de la superficie radicular.

\section{Tratamiento del conducto radicular}

Previa anestesia troncular infraorbitaria del lado izquierdo, se realizó aislamiento y apertura coronal, se estimó la longitud de trabajo en $20 \mathrm{~mm}$, conducto amplio, se instrumentó con el sistema Protaper hasta la lima F5, acompañada de irrigación con hipoclorito de sodio al 5,25\%, y remoción del smear layer con $3 \mathrm{ml}$ de quelante líquido para endodoncia 
EDTA al 1,7\% $\left(\right.$ Eufar $\left.^{\circledR}\right)$, dejándolo intraconducto por tres minutos, seguido de irrigación con hipoclorito de sodio al 5,25\% dejándolo dentro del conducto por 5 minutos. Posteriormente, el conducto fue obturado con un apósito de hidróxido de calcio en pasta, Calcifar - P $\left(\right.$ Eufar $^{\circledR}$ ) (Figura 2 y 3). ${ }^{21-33}$

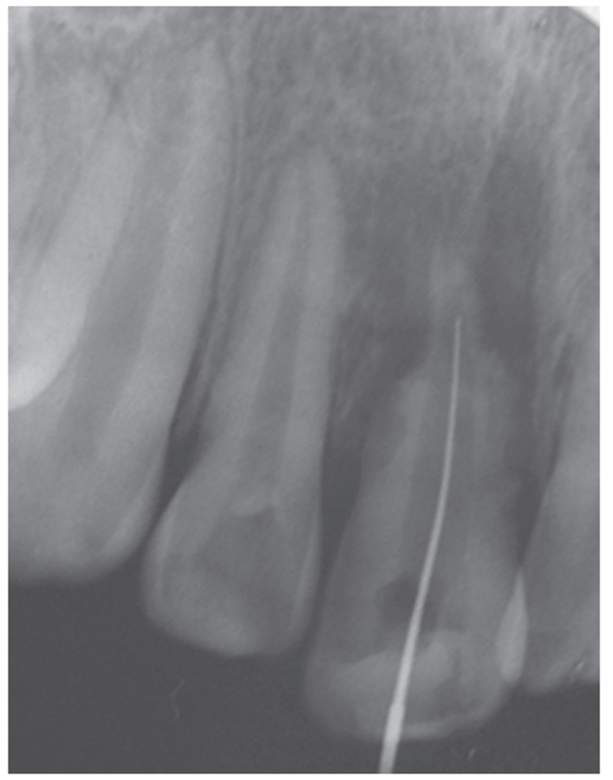

Figura 2. Estimación de la longitud de trabajo en $20 \mathrm{~mm}$.

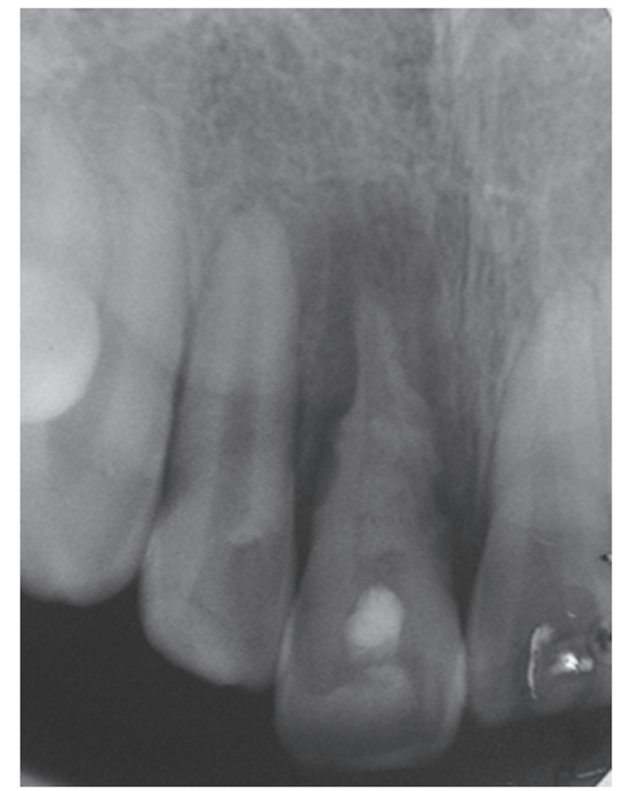

Figura 3. Dressing de hidróxido de calcio. Control radiográfico a los seis meses. Se observó disminución de la lesión radiolúcida.

Se realizaron recambios del apósito cada cuatro semanas por un período de seis meses. Sin embargo, al cabo de este tiempo los resultados obtenidos en la valoración clínica y radiográfica mostraban una resolución parcial de la patología.
Examen clínico:

- Los tejidos circundantes se observaban normales.

- Al realizar el sondaje se obtuvieron las mismas longitudes de profundidad de las bolsas periodontales.

- Dolor a la percusión.

- No se observaron cambios de color en la corona del diente.

- Movilidad dentaria grado II.

\section{Examen radiográfico:}

- Disminución de la lesión periapical.

- Persistencia del proceso reabsortivo.

- Persistencia de la pérdida ósea a nivel del hueso alveolar.

- Pérdida de la continuidad del ligamento periodontal.

Al realizar el control, a los ocho meses, el paciente refiere dolor a estímulos térmicos en el cuadrante superior izquierdo. Al realizar la valoración clínica se presentó dolor leve a la percusión en el diente 22, al realizar las pruebas de sensibilidad pulpar el diente 22 respondió positivamente y se logró reproducir la molestia manifestada por el paciente. El diagnóstico fue pulpitis irreversible del 22; por tal razón, se decidió realizar el tratamiento del conducto radicular del diente 22, en una sola cita (Figura 4).

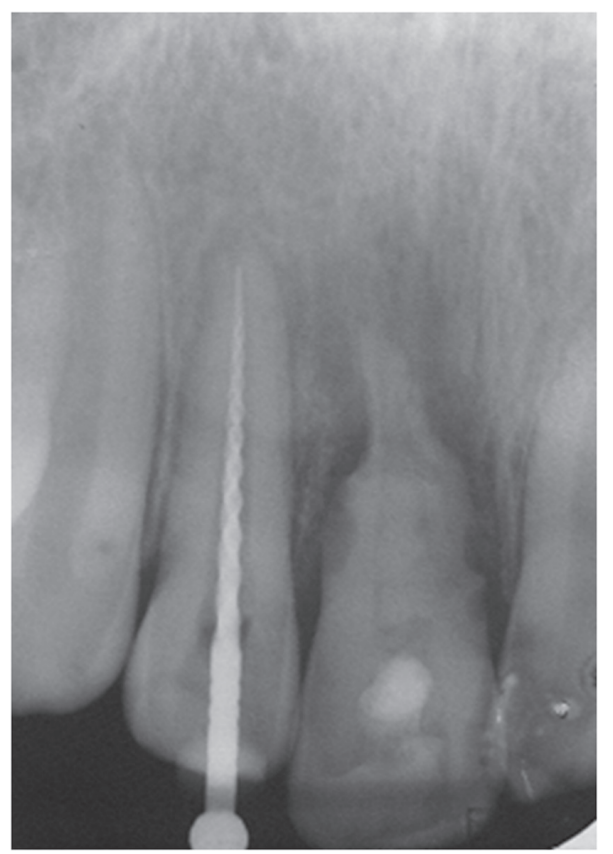

Figura 4. Dressing de hidróxido de calcio. Control radiográfico a los 8 meses. Marcada disminución de la lesión radiolúcida periapical del diente 21. Persistían las lagunas de reabsorción en la superficie radicular. Inicio del tratamiento de conducto del diente 22 . 
Después de haber realizado estos procedimientos y al tener en cuenta la respuesta parcial al tratamiento realizado, se decidió obturar completamente el conducto radicular del diente 21 con MTA blanco (Angelus ${ }^{\circledR}$ ) (Figura 5 y 6).

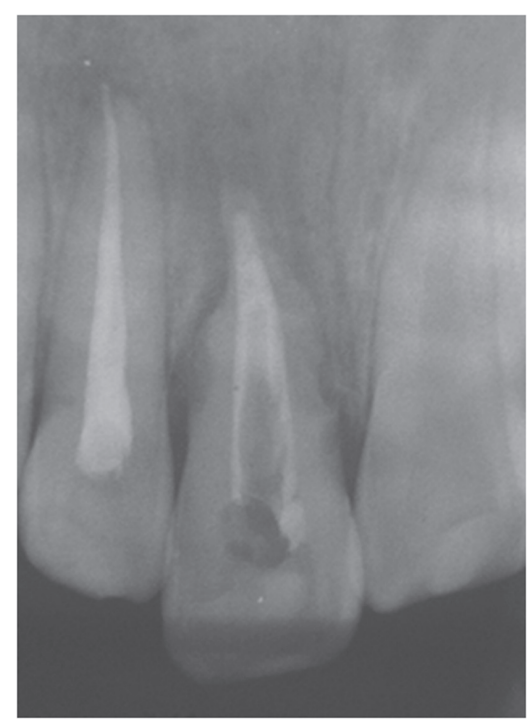

Figura 5. Tratamiento terminado del conducto radicular del diente 22 , conducto radicular del diente 21 con MTA.

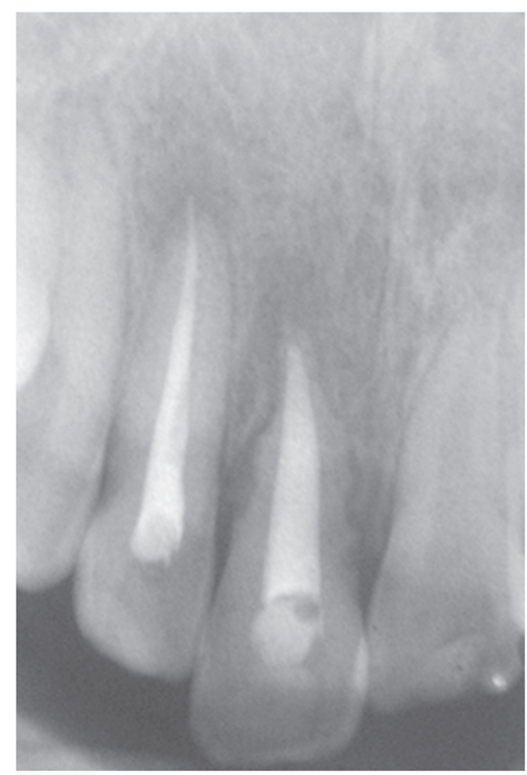

Figura 6. Obturación completa del conducto radicular del diente 21 con MTA.

En la radiografia de control tomada a los 24 meses, se observó una avanzada reparación de la lesión periapical y cambios a nivel del hueso alveolar - cresta alveolar y trabeculado en las superficies mesial y distal (formación ósea) y detención de la reabsorción.
Los controles radiográficos a los 48 y 54 meses (Figura 7 y 8) revelaron una completa reparación de la lesión periapical, de igual manera se observó una detención de la reabsorción y cambios en la densidad ósea, en el trabeculado y en la lámina ósea alrededor de la superficie radicular. Era observable una continuidad del ligamento periodontal alrededor de la superficie radicular.

Clínicamente, no había sintomatología a la percusión, el diente presentó un grado I de movilidad; las pruebas periodontales al sondaje no provocaron sangrado, los valores que se obtuvieron fueron de $4 \mathrm{~mm}$ en la superficies mesial, distal, vestibular y palatina del diente 21 .

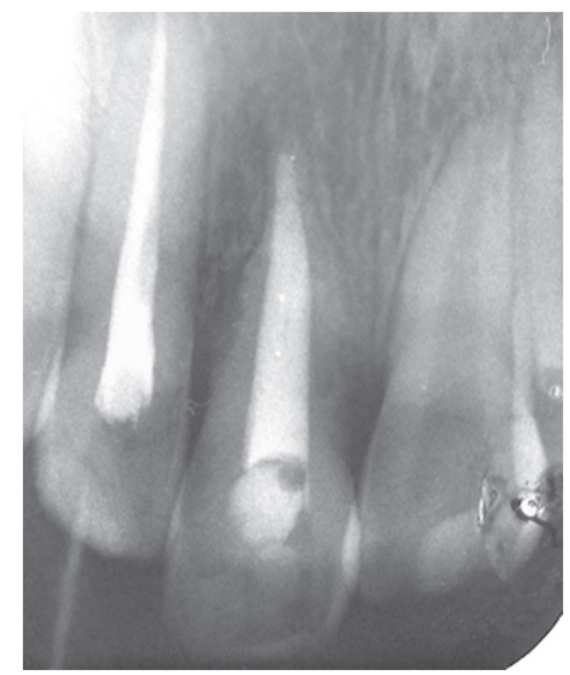

Figura 7. Control radiográfico a los 48 meses, se mantuvo la detención de la reabsorción. Se evidenció la reparación completa de la lesión periapical. Continúa la reparación del hueso alveolar - cresta alveolar y trabeculado en la superficies mesial y distal.

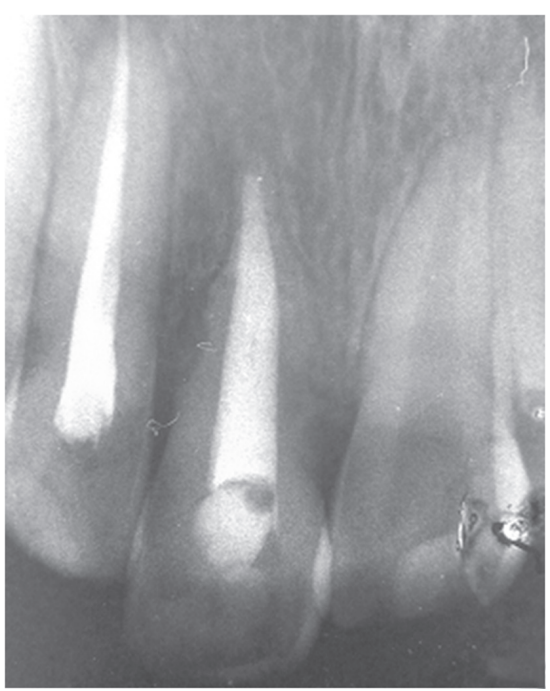

Figura 8. Control radiográfico a los 54 meses. Se mantuvo estable la condición clínica. 


\section{DISCUSIÓN}

Los factores más críticos que influyen en el éxito de un reimplante dental son el medio de transporte en el cual el diente avulsionado es colocado hasta que el paciente pueda ser valorado por un odontólogo y el tiempo extraoral, que se ha considerado como el período comprendido entre la avulsión del diente hasta su reimplante que, idealmente, no debería superar los 30 minutos. Después de la reimplantación debe aplicarse una férula semirrígida por un período de 7 a 10 días ya que se ha comprobado que una ferulización prolongada podría iniciar un proceso de anquilosis del diente. ${ }^{34-42}$

Las guías para el manejo de la avulsión de dientes permanentes con ápice cerrado, tiempo extraoral mayor de 60 minutos, mantenidos en un medio seco de transporte, indican: ${ }^{35,43-46}$

1. Eliminar el ligamento periodontal necrótico.

2. Eliminar el coágulo del alvéolo irrigándolo con suero fisiológico y examinarlo. Si hay una fractura en la pared del alvéolo, posicionarla de nuevo con un instrumento adecuado.

3. Sumergir el diente en una solución del fluoruro del sodio durante un mínimo de cinco minutos, reimplantarlo mediante presión digital suave.

4. Suturar la laceración gingival en el área cervical, verificar radiográficamente la posición normal del diente reimplantado y aplicar una férula flexible durante 4 a 6 semanas.

5. En los dientes con ápices cerrados, se debe realizar el tratamiento endodóntico después de dos semanas para prevenir el riesgo de reabsorción inflamatoria de la superficie radicular.

6. Administrar antibióticos: doxiciclina o penicilina por 7 días en las dosis adecuadas de acuerdo con la edad y el peso del paciente.

7. Referir al paciente a su médico para evaluar las necesidades de vacunación antitetánica, si el diente avulsionado ha estado en contacto con el suelo o si la cobertura de la vacuna antitetánica es incierta.

8. Las instrucciones para el paciente son: seguir una dieta blanda durante dos semanas, cepillado de los dientes con un cepillo suave después de cada comida y el uso de un enjuague bucal con clorhexidina al $0,12 \%$ dos veces al día durante siete días.

9. Realizar semanalmente los controles clínicos y radiográficos entre la primera y la octava semana. Posteriormente a los seis meses y anualmente hasta completar los 5 años de seguimiento.
Con respecto a la cicatrización periodontal, se han descrito tres diferentes tipos de reabsorción radicular externa inflamatoria post traumática: ${ }^{2,47,48}$

1. Superficie de reabsorción (reabsorción radicular relacionada con reparación).

2. Reabsorción inflamatoria (reabsorción radicular relacionada con infección) también llamada reabsorción radicular por infección pulpar o en los casos de estímulo bacteriano originado en el espacio periodontal (reabsorción radicular por infección periodontal).

3. Reabsorción por reemplazo (reabsorción radicular relacionada con anquilosis).

Estudios clínicos y experimentales han mostrado que el hidróxido de calcio induce la cicatrización periapical, ${ }^{49-52}$ elimina la flora microbiana presente en el conducto radicular, ${ }^{53-55}$ y detiene la reabsorción radicular inflamatoria. ${ }^{56-62}$

Adicionalmente, el Trióxido de Mineral Agregado (MTA) ha sido propuesto como un material adecuado para la apexificación en una sola cita, combina la biocompatibilidad y una acción bacteriostática con una favorable habilidad de sellado cuando es usado para reparar perforaciones radiculares o de la cámara pulpar y promueve la regeneración de la pulpa dental y del tejido periapical. ${ }^{62-70}$ También, es usado como material de obturación apical. ${ }^{63,71-73}$

El MTA ofrece una barrera en la porción apical del conducto (tapón apical) en dientes con pulpas necróticas y ápices abiertos aplicable a casos donde la constricción apical no existe. ${ }^{63}$

El pronóstico del trauma dental es incierto. En este reporte de caso se usó el MTA como una opción alternativa de tratamiento con un válido enfoque que permitió una larga supervivencia del diente. Las condiciones estéticas, funcionales y protésicas fueron preservadas y se evitó la aplicación de una prótesis dental de manera temprana en un paciente joven.

Aunque este reporte de caso presenta un resultado favorable, es necesario realizar estudios adicionales para soportar el uso de MTA en los casos de reabsorción radicular externa inflamatoria.

\section{BIBLIOGRAFÍA}

4. Tronstad L. Root resorption-etiology, terminology and clinical manifestations. Endod Dent Traumatol 1988; 4: $241-252$.

5. Ne RF. Tooth resorption. Quintessence Int 1999; 30: 9 - 25. 
6. Andreasen JO, Andreasen FM, Bakland LK, Flores MT. Traumatic Dental Injuries - A Manual. Copenhagen: Munksgaard; 1999. p. 9 - 11.

7. Trope M, Yesiloy C, Koren L, Moshonov J, Friedman S.Effect of different endodontic treatment protocols on periodontal repair and root resorption of replanted dog teeth. J Endod 1992; 18: 492 - 496.

8. Panzarini SR Gulinelli JL, Poi WR, Sonoda CK, Pedrini D, Brandini DA. Treatment of root surface in delayed tooth replantation: a review of literature. Dental Traumatol 2008; 24: 277 - 282.

9. Andreasen JO, Borum MK, Jacobsen HL, Andreasen FM. Replantation of 400 avulsed permanent incisors 4. Factors related to periodontal ligament healing. Endod Dent Traumatol 1995; 11: 76 - 89.

10. Hammarstrom L, Blomof L, Lindskog S. Dynamics of dentoalveolar ankylosis and associated root resorption. Endod Dent Traumatol 1989; 5: 163 - 175.

11. Wallace JA, Vergona K. Epithelial rests function in replantation: is splinting necessary in replantation? Oral Surg Oral Med Oral Pathol 1990; 70: 644 - 649.

12. Lindskog S, Blomlof L. Mineralized tissue formation in periodontal wound healing. J Clin Periodontol 1992; 19: $741-748$.

13. Harmmarstrom L. Enamel matrix, cementum development and regeneration. J Clin Periodontol 1997; 24: 658 -668 .

14. Trope M, Moshonov J, Nissan R, Buxt P, Yesilsoy C. Short vs. long-term calcium hydroxide treatment of established inflammatory root resorption in replanted dog teeth. Endod Dent Traumatol 1995; 11: $124-128$.

15. Hupp JG, Mesaros SV, Aukhil I, Trope M. Periodontal ligament vitality and histologic healing of teeth stored for extended periods before transplantation. Endod Dent Traumatol 1998; 14: $79-83$.

16. Blomlof L. Storage of human periodontal ligament cells in a combination of different media. J Dent Res 1981; 60: $1904-1906$.

17. Ashkenazi M, Sarnat H, Keila S. In vitro viability, mitogenicity and clonogenic capacity of periodontal ligament cells after storage in six different media. Endod Dent Traumatol 1999; 15:149 - 156

18. Andreasen JO. Effect of extra-alveolar period and storage media upon periodontal and pulpal healing after replantation of mature permanent incisors in monkeys. Int $\mathrm{J}$ Oral Surg1981; 10: $43-53$.

19. Andreasen JO. The effect of pulp extripation or root canal treatment on periodontal healing after replantation of permanent incisors in monkeys. J Endod 1981; 7: 245 $-252$.

20. Andreasen JO, Borum M, Jacobsen HL, Andreasen FM. Reimplantation of 400 avulsed permanent incisors. 2. Factors related to pulp healing. Endod Dent Traumatol 1995; 11: $59-68$.

21. Okamoto T, Hanada E, Saad Neto M. Reimplantation of incisor teeth of rats: the importance of nonvital cementum periodontal ligament. Rev Odontol UNESP 1986; 16: $53-64$.

22. Pohl Y, Filippi A, Kirschner H. Results after replantation of avulsed permanent teeth. II. Periodontal healing and the role of physiologic storage and antiresorptive-regenerative therapy. Dent Traumatol 2005; 21: $93-101$.

23. Blomlof L, Andersson L, Lindskog S, Hedstrom KG, Ha- mmarstrom L. Periodontal healing of replanted monkey teeth prevent from drying. Acta Odontol Scand 1983; 41: $117-123$.

24. Trope M. Root resorption of dental and traumatic origin. Classification based on etiology. Pract Periodontics Aesthet Dent 1998; 10: 515 - 522.

25. Baumgartner JC, Cuenin PR. Efficacy of several concentrations of sodium hypochlorite for root canal irrigation. J Endod 1992; 18: 605 - 612.

26. Bystrom A, Sundqvist G. Bacteriologic evaluation of the efficacy of mechanical root canal instrumentation in endodontic therapy. Scand J Dent Res 1981; 89: 321 - 328.

27. Bystrom A, Sundqvist G. The antibacterial action of sodium hypochlorite and EDTA in 60 cases of endodontic therapy. Int Endod J 1985; 18: 35 - 40.

28. Peters LB, van Winkelhoff AJ, Buijs JF, Wesselink PR. Effects of instrumentation, irrigation and dressing with calcium hydroxide on infection in pulpless teeth with periapical bone lesions. Int Endod J 2002; 35: 13 - 21.

29. Pérez-Heredia M, Ferrer-Luque CM, González-Rodríguez MP, Martín-Peinado FJ, González-López S. Decalcifying effect of 15\% EDTA, 15\% citric acid,5\% phosphoric acid and $2.5 \%$ sodium hypochlorite on root canal dentine. Int Endod J 2008; 41: 418 - 423.

30. Dogan H, Calt S. Effects of chelating agents and sodium hypochlorite on mineral content of root dentin. J Endod 2001; 27: 578 - 580 .

31. Zehnder M. Root canal irrigants. J Endod 2006; 32: 389 - 398.

32. Bystrom A, Happonen RP, Sjogren U, Sundquvist G. Healing of periapical lesion of pulpless teeth after endodontic treatment with controlled asepsis. Endod Dent Traumatol 1987; 3: 58 - 83 .

33. Shah N. Nonsurgical management of periapical lesions: a prospective study. Oral Surg Oral Med Oral Pathol 1988; 66: 365 - 371.

34. Çaliskan MK,Sen BH. Endodontic treatment of teeth with apical periodontitis using calcium hydroxide: a long-term study. Endod Dent Traumatol 1996; 12: 215 - 221.

35. Çaliskan MK, Türkün M. Periapical repair and apical closure of a pulpless tooth using calcium hydroxide. Oral Surg Oral Med Oral Pathol 1997; 84: 683 - 686.

36. Sjögren U, Figdor S, Spångberg L, Sundqvist G. The antimicrobial effect of calcium hydroxide as a short-term intracanal dressing. Int Endod J 1991; 24: 119 - 125.

37. Andreasen JO, Andreasen FM Essentials of Traumatic Injuries to the Teeth, 2nd Ed. Copenhagen: Munksgaard Mosby; 2000. p. $113-131$.

38. Flores MT, Andersson L, Andreasen JO, Bakland LK, Malmgren B, Barnett F. Guidelines for the management of traumatic dental injuries. II. Avulsion of permanent teeth. Dent Traumatol 2007; 23: 130 - 136.

39. Trope M. Current concepts in the replantation of avulsed teeth. Alpha Omegan 1997; 90: 56 - 63.

40. Andreasen JO, Andreasen FM. Avulsions. Textbook and Color Atlas of Traumatic Injuries to the Teeth. 3rd Edition St Louis: Mosby; 1994. p. 383-425.

41. Pohl Y, Wahl G, Filippi A, Kirschner H. Results after replantation of avulsed permanent teeth. III. Tooth loss and survival analysis. Dent Traumatol 2005; 21: 102 - 110.

42. Pohl Y, Tekin U, Boll M, Filippi A, Kirschner H. Investigation on a cell culture medium for storage and transpor- 
tation of avulsed teeth. Aust Endod J 1999; 25: 70 - 75.

43. Funicane D, Kinirons MJ. External inflammatory and replacement resorption of luxated and avulsed replanted permanent incisors: a review and case presentation. Dent Traumatol 2003; 3: 170 - 174.

44. Malmgren B, Malmgren O. Rate of infraposition of reimplanted ankylosed incisors related to age and growth in children and adolescents. Dent Traumatol 2002; 18: 28 -36 .

45. Tzigkounakis V, Merglova V, Hecova H, Netolicky J. Retrospective clinical study of 90 avulsed permanent teeth in 58 children. Dent Traumatol 2008; 24: $598-602$.

46. Trope M. Clinical management of the avulsed tooth: present strategies and future directions. Dent Traumatol 2001; 18: $1-11$.

47. Majorana A, Bardelini E, Conti G, Keller E, Passini S. Root resorption in dental trauma: 45 cases followed for 5 years. Dent Traumatol 2003; 19: 262 -265.

48. Robertson A, Robertson S, Nore'n JG. A retrospective evaluation of traumatized permanent teeth. Int J Paediatr Dent 1997; 7: 217 - 226.

49. American Association of Endodontits. Treatment of the avulsed permanent tooth. Recommended guidelines of the American Association of Endodontists. Dent Clin North Am 1995; 39: 221 - 225.

50. Chappuis V, Von Arx T. Replantation of 45 avulsed permanent teeth: a 1-year follow-up study. Dent Traumatol 2005; 21: $289-296$.

51. Fuss Z, Tsesis I, Lin S. Root resorption - diagnosis, classification and treatment choices based on stimulation factors. Dent Traumatol 2003; 19: $175-182$.

52. Cvek M. Treatment of non-vital permanent incisors with calcium hydroxide. Part I. Follow-up of periapical repair and apical closure of immature roots. Odontol Revy 1972; 23: 27 - 44 .

53. Kerekes K, Heide S, Jacobsen I. Follow-up examination of endodontic treatment in traumatized juvenile incisors. J Endod 1980; 6: 744 - 748 .

54. Cvek M. Prognosis of luxated non-vital maxillary incisors treated with calcium hydroxide and filled with guttapercha. A retrospective study. Endod Dent Traumatol 1992; 8: 45 - 55 .

55. Mackie IC, Worthington HV, Hill FJ. A follow-up study of incisor teeth which had been treated by apical closure and root filling. Br DentJ 1993; 175: 99 - 101.

56. Bystrom A, Claesson R, Sundqvist G.The antibacterial effect of camphorated paramonochlorophenol, camphorated phenol and calcium hydroxide in the treatment of infected root canals. Endod DentTraumatol 1985; 1: 170 $-175$.

57. Bystrom A, Haaponen RP, Sjogren U, Sundqvist G. Healing of periapical lesions of pulpless teeth after endodontic treatment with controlled asepsis. Endod Dent Traumatol 1987; 3: 58 - 63.

58. Sjogren U, Figdor D, Spangberg L, Sundqvist G.The antimicrobial effect of calcium hydroxide as a short term intracanal dressing. Int Endod J 1991; 24: 119 - 125.

59. Cvek M.Treatment of non-vital permanent incisors. Part II. Effect on external root resorption in luxated teeth compared with the effect of root filling with gutta percha. Odontol Revy 1973; 24: 343 -354

60. Hammarstrom LE, Blomlof LB, Feiglin B, Lindskog SF.
Effect of calcium hydroxide treatment on periodontal repair and root resorption. Endod DentTraumatol 1986; 2: $184-189$.

61. Trope M, Yesilsoy C, Koren L, Moshononv J, Friedman S. Effect of different endodontic treatment protocols on periodontal repair and root resorption of replanted dog teeth. J Endod 1992; 18: 492 -496.

62. Trope M, Moshonov J, Nissan R, Buxt P, Yesilsoy C. Short vs.long-term calcium hydroxide treatment of established inflammatory root resorption in replanted dog teeth. Endod DentTraumatol 1995; 11: 124 - 128.

63. Tronstad L, Andreasen JO, Hasselgren G, Kristerson L, Riis I. pH changes in dental tissues after root canal filling with calcium hydroxide. J Endod 1980; 7: 17 - 21.

64. Cvek M. Prognosis of luxated non-vital maxillary incisors treated with calcium hydroxide and filled with guttapercha. A retrospective study. Endod Dent Traumatol 1992; 8: 45 - 55.

65. Tronstad L. Pulp reactions in traumatized teeth. En: Gutmann JL, HarrisonJW. Proceedings of the International Conference on Oral Trauma. Chicago: American Association of Endodontists Endowment \& Memorial Foundation; 1986. p. 55 - 78.

66. Torabinejad M, Chiavian N. Clinical application of mineral trioxide aggregate. J Endod 1993; 25: 197 - 205.

67. Shabahang $S$, Torabinejad M. Treatment of teeth with open apices using mineral trioxide aggregate. Pract Periodontics Aesthet Dent 2000; 12: 315 - 320.

68. Maroto M, Barberia E, Planells P, Vera V. Treatment of a non-vital immature incisor with mineral trioxide aggregate (MTA). Dent Traumatol 2003; 19: 217 - 221.

69. Koh ET, McDonald F, Pitt Ford TR, Torabinejad M. Cellular response to mineral trioxide aggregate. J Endod 1998; 24: $543-547$.

70. Holland R, de Souza V, Nery MJ, Otoboni Filho JA, Bernabe $\mathrm{PF}$, Dezan Junior E. Reaction of rat connective tissue to implanted dentin tubes filled with mineral trioxide aggregate or calcium hydroxide. J Endod 1999; 25: 161 - 166.

71. Torabinejad M, Hong CU, Pitt Ford TR, Kettering JD. Antibacterial effects of some root end filling materials. J Endod 1995; 21: 403 - 406.

72. Arens DE, Torabinejad M. Repair of furcal perforations with mineral trioxide aggregate: two case reports. Oral Surg Oral Med Oral Pathol Endod Radiol 1996; 82: 84 - 88.

73. Holland R, Filho JA, de Souza V, Nery MJ, Bernabe PF, Junior ED. Mineral trioxide aggregate repair of lateral root perforations. J Endod 2001; 27: 281 - 284.

74. Menezes R, Bramante CM, Letra A, Carvalho VG, Garcia RB. Histologic evaluation of pulpotomies in dog using two types of mineral trioxide aggregate and regular and White Portland cements as wound dressings. Oral Surg Oral Med Oral Pathol Oral Radiol Endod 2004; 98: 376 379.

75. Torabinejad M, Smith PW, Kettering JD, Pitt Ford TR. Comparative investigation of marginal adaptation of mineral trioxide aggregate and other commonly used rootend filling materials. J Endod 1995; 21: 295 - 299.

76. Xavier CB, Weismann R, de Oliveira MG, Demarco FF, Pozza DH. Root-end filling materials: apical microleakage and marginal adaptation. J Endod 2005; 31: 539 - 542. 